\title{
Effects of music on driver behavior
}

\author{
ZAID MAHMOUD \\ Department of transport telematics \\ Czech Technical University in Prague \\ mahmozai@fd.cvut.cz \\ CZECH REPUBLIC \\ MARTIN RUZEK \\ Department of Intelligent transportation systems \\ Czech Technical University in Prague \\ martin.ruzek@cvut.cz \\ CZECH REPUBLIC
}

\begin{abstract}
The attention level of car drivers is affected by many factors. Music is one of the most important ones, but its effect is rarely studied. Music can affect driving style in both positive and negative ways, as it can reduce fatigue but also increase the level of distraction or aggression. This article presents an experimental investigation of the effects of music on driver attention level. Several measurements on a vehicle simulator were done to collect data that demonstrates the relationship between music and the performance of the car driver. The simulation measured performance under three conditions - relaxation music, rock music and silence. Additionally, the measurements were repeated in both fresh and tired states. The results are, in some aspects, different from our expectations - for example, relaxation music improved reaction time but also correlated with a higher occurrence of inappropriate steering actions. Deeper understanding of how the music and noise affect the driver's actions and decisions will help to improve road safety and reduce the probability of accidents.
\end{abstract}

Key-Words: - music, driving, fatigue, vehicle, simulator

Received: April 26, 2020. Revised: August 18, 2020. Accepted: September 26, 2020. Published: October 30, 2020.

\section{Introduction}

\subsection{Motivation}

The human factor is the least reliable part of any transport system and, in the case of road traffic, driver's mistakes are the principal cause of fatal accidents. Deeper understanding of the factors that influence driver behavior would help to design systems that increase safety by predicting human faults.

Music is definitely an important factor concerning the behavior of a driver [17]; however, its impact is rarely subject to investigation. The reason for this is that the influence of music is quite complicated and highly individual. The same type of music can have a relaxing effect on one person while having the complete opposite effect on another. This study aims to learn more about this phenomenon by the use of statistical methods.

\subsection{Relevant research}

The vehicle simulators are widely used to study the driver's responses to various situations [13], but music-related research is relatively scarce. The existing studies related to the effect of music on drivers vary in their measurement methods and algorithms. The impact of music's effect on a driver alternates between positive and negative. Some studies consider listening to music as a distractor while others have found it to improve driver performance.

Wang and colleagues [1] examined 165 university students participating in two driving tests (with and without music) of twelve minutes for each drive. The first half of both driving scenarios started with a light driving situation (countryside) with the simple objective of following a lead car. The second half of both scenarios (music and no music) simulated more complicated driving situations, (city driving) with 
more complicated driving obstacles and more braking required.

This study, when compared with the results of other similar studies and research [2], confirmed that music improved the car-following delay. This research also found, as did other research [3], that higher tempo music leads to more un-respected traffic lights, more accidents, and more lane crossing.

The authors, in general, mentioned that music had no clear effect on simple driving situations (neither positive nor negative) while affirming that music contributed to negative consequences in more complex driving situations. The authors added that this could be a reason why the results differ from one driving situation to another.

Brian H. Dalton and David G. Behm [4] have presented a comprehensive systematic review that includes numerous studies about the role of noise and music on human performance. The authors studied the effects of music on general human tasks, including driving tasks. The study was divided into four parts: the first part focused on fresh driver and music-related studies; the second part focused on the effects of background music on driver stress and anxiety; the third part focused on background music tempo and driving performance; the last part focused on background music and driving performance.

With respect to the first part concerning fresh drivers and music studies, the first studies related to driving while listening to background radio sound started in the 1960s [5-6]. Brown, the conductor of the first study, reported that music reduced brake usage in light traffic. Although he also found that music in heavy traffic increased the time to finish the course and served as a distractor, it also reduced the amount of stress and emotional arousal present while driving. In a second study, S. Konz and D. McDougal [6] compared control activities (steering wheel movements, brake usage, and accelerator usage) while listening to silence, slow music, and Tijuana brass music in the background. The results showed a difference when both types of music were in the background. It was hard to distinguish if the change from the control activities was good or bad. However, unlike in the first study, the presence of music decreased the amount of time which was required to finish the course as there seemed to be an excitement factor which increased the speed while listening to music.

In the second part concerning background music and how it affects driver stress and anxiety, the authors covered many studies and psychological and physiological aspects, such as studies which found that listening to music reduces stress [7] and blood pressure [8]. Many studies have focused on driver stress and many have found that it is hard to formalize the effects of music in light traffic. Certain studies affirmed that listening to music while the heavy traffic driving, especially the favorite music of the driver, can reduce the probability of an accident [911]. Brian H. Dalton and David G. Behm [4] suggest in their systematic review that drivers are less stressed and aggressive while listening to music due to its tendency to distract and decrease a driver's attention from the surrounding stressors.

An interesting comparison can be found in [12] where the authors suggest that listening to the radio talk helps the driver to stay focused, but listening to music has no effect on the performance.

Concerning the third part, background music tempo and driving performance, the authors pointed out that there have not been many studies which focus on the tempo of background music. Generally, results are conflicted when determining whether tempo has a positive or negative effect on a driver's behavior. However, in a study by Brodsky [3], the participants underestimated their own speed while listening to higher tempo music while also suffering from more accidents and more passed red lights.

In the last part concerning background music driving performance, equivocal results are presented. Matthews and his colleagues [14] found that listening to medium intensity hard rock improved performance, but not high intensity.

In contrast to the studies that report music as having no influence on driver behavior during light stress driving, there are some studies which report the opposite. Beh and Hirst [15] found that music did not help ease performance during simple tracking tasks which required vasomotor coordination and continuous motor involvement.

We can see from these studies that the effects of music while driving are still equivocal, therefore, in our study, we evaluated the effects of music on driver performance via numerous measuring methods such as eye-tracking, response time and vehicle control (Following a lead car and car position on the road). We covered different driver situations as well, such as a normal subject situation versus a fatigued subject situation. Each subject listened to two types of music (Rock, Classical) in addition to a period of silence. The objective was to determine whether the music could have any benefits on driving alone on the highway.

\section{Methodology}

The main problem concerning the study of the human driver is its huge complexity as well as the complicated relationship between input and output. To study the effect music has on this process, the 
optimal method would be to eliminate all other factors, such as hours of the last sleep, glucose level, etc. This is, however, very difficult to achieve in reality. The solution to this problem is a statistical approach. We do not try to repeat the measurements under the same conditions, nor to start every measurement from the same state, but rather to repeat the measurements multiple times and record the related condition during the experiment. In short, the measurements are repeated in a fresh and tired state and with different music sequences.

The available test bench enables the measurement of two types of data: physiological data and technical data.

Physiological data come directly from the driver's body. Typical examples of physiological data are those from the EEG, eye tracker, heart rate monitor, etc. However, a disadvantage is that these data are strongly affected by noise. In the simulator used for this study, only the eye tracker was available.

The technical data which came from the simulator was divided into 2 subgroups: vehicle data and driving data.

The vehicle data are related to the position of the vehicle, further vehicle data includes vehicle speed, lateral position or distance to the lead vehicle, etc.

The driving data are the commands given by the driver to the vehicle, such as movements of the steering wheel or pushing of the pedals. The driving data are correlated with the vehicle data- for example, if the driver steers, the vehicle position changes. However, if the steering is fast and short, the trajectory of the vehicle will not be affected. This rapid and small movement of the steering wheel can be a sign of increasing fatigue.

\subsection{Physiological data}

The eye tracker is a device that measures the state of the eyelids and the point of fixation of the eye [16]. The following data were extracted from the eye tracker output:

a) Blink interval - the time between 2 consecutive eye blinks. Low blink interval means high blinking frequency. High blink interval is typical for fatigue.

b) Fixation duration - the time when the eyes of the driver are open and fixed onto one point without any movement. A long time of fixation duration is more common during the somnolence. c) Saccade interval - the time between two consecutive movements of the eyes. Low saccade interval means more agile movements of the eyes.

\subsection{Vehicle data}

The technical indicators are achieved by analysis of the driving data from the simulator, in short, of lateral control, longitudinal control and reaction time.

a) Lateral control is the distance from the center of the lane. The driver should follow the ideal trajectory, regardless of the lead vehicle.

b) Longitudinal control is the time distance between the lead vehicle and the simulated vehicle. The task is to follow the lead car as close as possible, but to avoid an accident. The lead car is changing its speed and position in the lane as well. The driver should adapt the speed, but always follow the ideal trajectory.

c) Reaction time is the time between the appearance of a stimulus on the screen and the reaction of the driver. The stimulus is a small red circle in the visual field which is triggered at random instances. If the driver was braking before the braking request, this event is then considered as invalid and the braking is not evaluated. If the reaction time is greater than three seconds, it is assumed that the driver took no reaction.

\subsection{Driving data}

The driver is giving commands to the vehicle by moving the steering wheel and pushing the pedals. Some of these commands can be very small and not observable at the vehicle level, but are still a good indicator of increasing fatigue or aggression [18].

In the scope of this research, two types of driving data were collected - fast movement of the steering wheel and fast braking.

Fast steering was defined as the steering with an angular speed of more than 80 degrees per second for a time of at least 320 milliseconds. The simulated road was almost straight, therefore no fast steering was needed.

Fast braking was defined as movement of the brake 
pedal with speed greater than 500 units per second (the position of the brake pedal is evaluated in the scale of 1 to 1000 units) for a minimum time of 200 milliseconds. Since the driver was sometimes requested to brake (in the moments when the red circle appeared), these events were excluded from the driving data evaluation. The reason for this is that in such moments, fast braking was correct and cannot be considered a mistake.

\subsection{Music environment}

During the test, the driver was exposed to different music types, which always fell into one of the following categories: relaxation music, rock music or silence. The duration of these music segments was approximately 15 minutes. The order of the music type changed in order to avoid the influence of a natural increase in fatigue. Therefore, for example, in one test the driver listened to a sequence of rock relaxation music - silence, while in another test to a sequence of silence - rock - relaxation.

To minimize the influence of other external conditions (such as hours of sleep before measurement, glucose level, etc.), the measurements had to be repeated at least three times.

\subsection{Simulator}

In the described scenario, the driver is driving on a highway at night. The task is to follow the lead car at a distance of fewer than 20 meters. We selected this scenario because according to our previous experience with the vehicle simulators, the situation with a lack of other stimuli is more convenient for the data evaluation. In more complex scenarios, it is also important to consider the actual situation ffor example, red vs. green traffic lights), which makes the analysis more complicated.

The lead car changes its speed and position in the lane.

The scenery is projected onto three screens, making an approximately $270^{\circ}$ panorama for the driver. The screens are approximately three meters from the driver. The simulator is equipped with motion control, therefore, acceleration or vibrations are not part of the simulation.

The simulator stores the driving data, such as the position of the vehicle, its speed, position of the steering wheel or brake pedal, etc. The time resolution of the vehicle simulator is 8 milliseconds.

The simulator is equipped with an eye tracker that has four infrared cameras. The eye tracker measures the eye position and movement of the driver. The time resolution of the eye tracker is 16 milliseconds.

\subsection{Participants}

The test was divided into two parts.

In the first part, two drivers were studied at a detailed level. Both test subjects were healthy men. The driving test lasted 45 minutes and was repeated three times in both fresh and tired states. The tired state is defined as a state with three hours or more of previous driving.

In the second part, a control group of 23-test drivers were subjected to driving in the same scenario. The test group consisted of 20 men, 3 women aged 18 to 36 years, and 1 to 11 years of driving experience. Each drive lasted for 120 minutes and one of the following music types were presented: silence, familiar music, death metal music and alpha waves (noise, sounds for relaxation). The category of familiar music was a music playlist which was selected by the subject him/herself, and we assumed it to have an identical effect as the relaxation music from the first part of the experiment.

In all cases, the simulated scenario was a nighttime drive on a highway with no traffic.

\section{Results}

\subsection{Eye tracker evaluation}

The movement of the eye is highly individual, therefore it is not possible to define a general method for all drivers. Despite this fact, we can observe certain dependencies in the behavior of the eye. In the scope of this research, the studied variables were the blink interval, fixation duration and, saccade interval.

The individual character of eye movement can be demonstrated by comparing a histogram of blink duration for two different drivers (Fig 1), which shows that driver B has a significantly higher blink frequency than driver $\mathrm{A}$ 


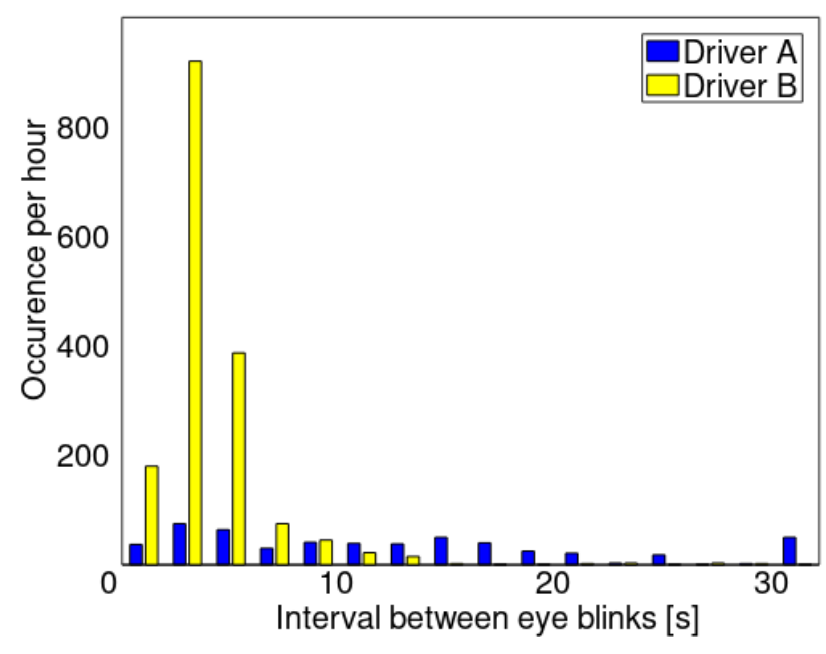

Fig. 1: Eye blink interval for 2 different drivers. The data is collected from 12 tests of 45 minutes.

With respect to eye fixation, driver A has higher values (longer fixation on one point) when listening to rock music, while for driver $\mathrm{B}$, the higher values occurred during silence. However, these differences are small (tables 1 and 2).

Saccade intervals have similar characteristics as fixation - for driver A, it is more common to increase this interval when listening to rock music, while for driver B, it was during silence.

In general, we can observe the impact of rock music on driver A (longer blink and saccade intervals and fixation duration), meaning his eyes are more stable. For driver B, the differences were less significant

\begin{tabular}{lll}
\hline & Driver A & Driver B \\
\hline Rock & 11.68 & 3.43 \\
\hline Silence & 7.28 & 3.8 \\
\hline Relaxation & 4.73 & 3.37 \\
\hline
\end{tabular}

Tab 1: Median of blink intervals for the individual drivers depending on music type [s]

\begin{tabular}{lcc}
\hline & Driver A & Driver B \\
\hline Rock & 0.76 & 0.76 \\
\hline Silence & 0.65 & 0.83 \\
\hline Relaxation & 0.58 & 0.68 \\
\hline
\end{tabular}

Tab 2: Median of eye fixation duration [s]

\begin{tabular}{lll}
\hline & Driver A & Driver B \\
\hline Rock & 0.78 & 0.71 \\
\hline Silence & 0.68 & 0.73 \\
\hline Relaxation & 0.60 & 0.63 \\
\hline
\end{tabular}

Tab 3: Median of saccade intervals for the individual drivers depending on music type [s]

\subsection{Evaluation of technical data}

\section{Lateral control}

For all three observed variables (lateral control, longitudinal control and reaction time), we observed relatively small dependency between music type and driver state.

With respect to the times when a driver drove out of lane (lane departure time), the time values seem quite different when both drivers are compared. However, it is important to mention that the moments of lane departure were quite rare (the highest occurrence is $75 \mathrm{~s}$ /hour for driver A and rock music). Therefore, these results have a low statistical value.

\begin{tabular}{lll}
\hline & Driver A & Driver B \\
\hline Rock & 1.03 & 0.32 \\
\hline Silence & 1.13 & 0.26 \\
\hline Relaxation & 1.07 & 0.27 \\
\hline
\end{tabular}

Tab 4. Average lateral deviation (distance from the center of the lane) in meters for both drivers exposed to different music types. 


\begin{tabular}{lll}
\hline & Driver A & Driver B \\
\hline Rock & 57 & 4 \\
\hline Silence & 45 & 0 \\
\hline Relaxation & 65 & 0 \\
\hline
\end{tabular}

Tab 5. Time spent out of lane for both drivers and all music types. The vehicle is considered as out of lane if the lateral distance to the ideal trajectory is more than $1.7 \mathrm{~m}$ ([time in seconds spent out of lane] per hour).

\section{Longitudinal control}

Longitudinal distance is defined as the time that a simulated vehicle spends out of the ideal position relative to the lead vehicle. The driver is asked to be as close to the lead vehicle as possible, without respecting the safety distance. Table 6 shows an evaluation of the times when longitudinal control exceeded a three second distance.

Interestingly, in a tired state this happened less frequently than in a fresh state, meaning that when tired, the drivers were following the lead car at a closer distance.

With respect to music, for driver A, the worst results were observed with relaxation music, while for driver $\mathrm{B}$ it was with rock music.

\begin{tabular}{lll}
\hline & Driver A & Driver B \\
\hline Rock & 18 & 38 \\
\hline Silence & 17 & 21 \\
\hline Relaxation & 23 & 6 \\
\hline
\end{tabular}

Tab 6. Time when the time distance between the lead and ego vehicle (vehicle where the tested person is sitting) was more than three seconds (in seconds per hour).

\section{Reaction time}

The reaction time was similar for all music states. In general, we observed slightly longer reaction times when listening to rock music in the tired state. As we noticed, the shortest reaction time was while driving fresh and listening to Rock music.

\section{Driver A Driver B}

Fresh Tired Fresh Tired

\begin{tabular}{lcccc}
\hline Rock & $\mathbf{9 5 2}$ & 1104 & $\mathbf{7 9 2}$ & 928 \\
\hline Silence & 1120 & 968 & 880 & 896 \\
\hline Relaxation & 1016 & 984 & 888 & 880 \\
\hline
\end{tabular}

Tab 7. Reaction time for both drivers (in milliseconds).

\subsection{Evaluation of driving data}

Driving data seems to be the best indicator of the level of attention of a driver. The number of events is relatively high, therefore the statistical significance is greater. Also, it is easier to interpret the driving data, because we can associate it with a certain state. For example, when the vehicle leaves the lane, or the driver does not brake when needed, we know that he lost the attention.

With respect to fast steering, driver A rarely made this error while for driver B it was more frequent. The difference is most notable in the tired state during silence.

For fast braking, the numbers are similar for driver A but are visibly different for driver B. During periods of relaxation music, he was more likely to stamp on the brake pedal.

In general, driver B seems to be more affected by music than driver A. He commits fewer mistakes when listening to rock music. 


\begin{tabular}{lcccc}
\hline & Driver A & & Driver B \\
& Fresh & Tired & Fresh & Tired \\
& & & & \\
\hline Relaxation & 0,0 & 0,0 & 3.9 & 0,0 \\
\hline Silence & 2,0 & 0,0 & 3.9 & 0,0 \\
\hline \multirow{2}{*}{ Rock } & 2,0 & 0,0 & 3.8 & 0,0 \\
\hline
\end{tabular}

Tab. 8: Fast steering events. The units are events per hour. Fast steering was most common for driver B in a tired state during silence. Fast steering is a sign of increasing fatigue and slower reaction.

\begin{tabular}{lcccc}
\hline & Driver A & \multicolumn{2}{c}{ Driver B } \\
& Fresh & Tired & Fresh & Tired \\
& & & & \\
\hline Relaxation & 0 & 14 & 19 & 21 \\
\hline Silence & 0 & 13 & 19 & 8 \\
\hline Rock & 4 & 8 & 9.5 & 3 \\
\hline
\end{tabular}

Tab 9: Fast braking events (events per hour).

\subsection{Evaluation of the control group}

In this part of the experiment, we evaluated a relatively large group of drivers, but without going into deep details as in the previous part. The results are similar for all music types except for the category of familiar music, where the reactions were approximately $300 \mathrm{~ms}$ faster. This is different from the first group, where we didn't observe any faster reactions with the relaxation music. The relative difference with the other music types is roughly 30 $\%$, therefore we can consider it as a significant impact.

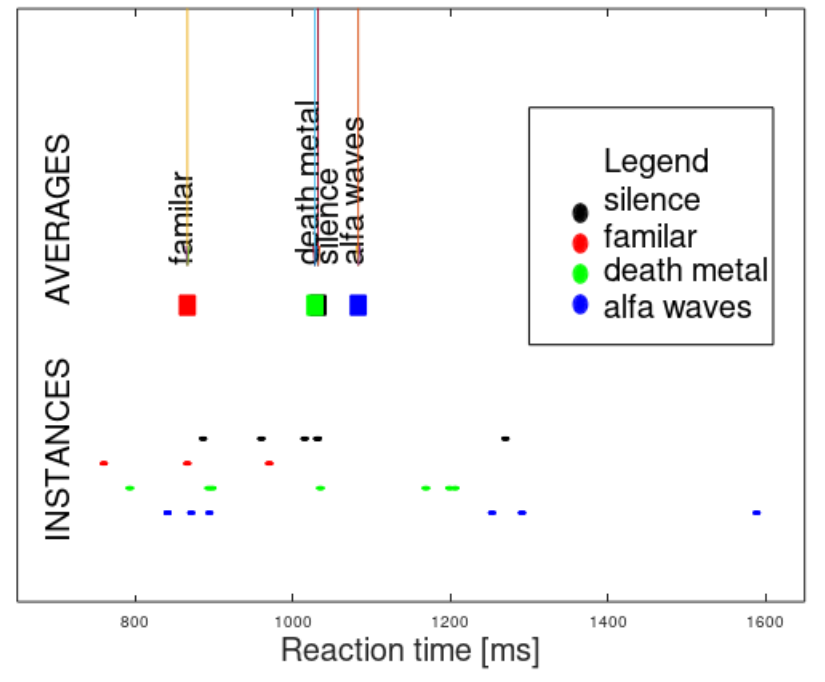

Fig 2. Reaction time for the control group of drivers exposed to different music types. The fastest reactions were during the "familiar" type of music. The upper part shows the aggregated values for the music type, the lower part shows the instances.

Secondly, we examined the changes in reaction time as the test progressed, to see if the reaction times at the beginning of the drive are different from in the later moments. The familiar music has slower reaction times during the whole 2 hours test. The periods of silence, death metal and alpha waves have similar scores. The worst reaction times were observed right at the beginning of the test. After 2 hours of driving, the results were almost the same. The results suggest that the effect of the music fades out with the increasing time when the driver listens to it. 


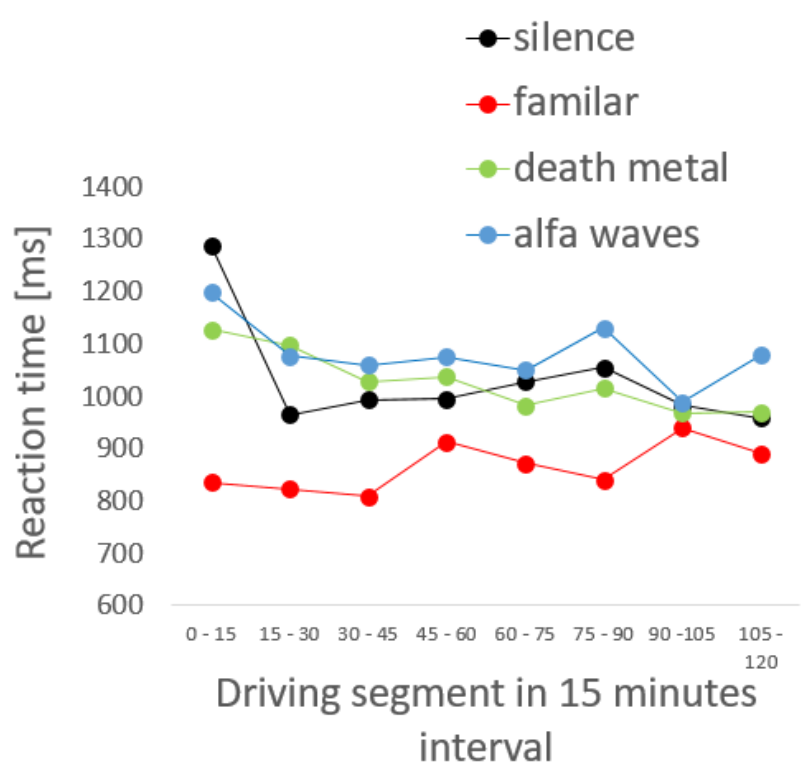

Fig 3: Change in reaction time during a 2 hour drive with different music styles.

\section{Discussion and future work}

One of the problems of this research is finding the right balance between the size of the tested group and the depth of testing. Reaction to music is highly individual; therefore, by averaging the results from a big test group we will lose the dependencies of an individual driver. On the other hand, for practical purposes it is important to achieve data from as many people as possible. For this reason, we chose a twolevel strategy: by in-depth testing of a small group of drivers, we tried to find dependencies and verify the results with a bigger test group. In the future, we would like to extend in-depth testing of a smaller group rather than increase the size of the verification test group.

For the first part (in-depth test of drivers) the results are significantly different for both drivers, which corresponds to the expectation that the influence of music on driver performance is highly individual.

The two main problems of the research are the interpretation of data and the filtration of irrelevant inputs.
The first problem is mostly visible in the case of physiological data. We can find certain correlations between music style and the eye tracker data, but we can hardly link them to any actual driver state. Of course, when the driver completely closes his eyes, we know that he has either fallen asleep or is at least not concentrated. This is, however, not a typical situation. Usually, we see only small differences in blinking frequency or fixation duration. It is an open question of what this means for driving.

The vehicle data is easily interpretable because, in the end, the position of the vehicle is the most important variable. The problem is that for this type of data we observed little difference. This is also expected because even a tired or distracted driver can control a vehicle without any notable mistakes. The 'big mistakes', such as leaving the lane or not reacting to a red light often happen without any previous warning. This fact limits the usage of vehicle data.

An important subgroup of the vehicle data is the driving data, which, in the scope of this research, were treated separately.

The main problem of all collected data is that they are affected by other inputs, not only by music. This makes taking proper measurements difficult and means that the experiments need to be repeated many times to minimize the influence of random error. This is planned for future investigations.

The obtained data confirmed the ambiguous effect of the music as reported by other relevant researches. Additionally, we studied the effects of the music on the low control level, such as the steering and braking actions, that showed up to be the most affected by the music. In our experiment, the negative effects of the music prevail the positive ones, but the effect is highly individual.

As the major benefit of the presented study we consider the quantity of observed variables during the experiment. Differently from the majority of other research projects, we collected both physiological and technical data. This 
makes the analysis more complex, but on the other hand, it brings deeper insight into what is happening in the driver's brain.

We noticed that the music has quite a fast effect on eye movements; however, this effect is hard to interpret. The effects on the vehicle position (which is, in the end, the most interesting variable) are quite small. The effects on the driving commands are more direct and, at least in the case of one tested person, we observed a negative effect of the music on the steering actions.

\section{Conclusions}

The observed data suggest that certain dependencies exist between music, on one hand, and driver behavior, on the other, although the nature of these dependencies is not straightforward. We tested two drivers in-depth, one showed little dependency on music while the other proved that relaxation music is actually not relaxing him, as his driving performance dropped during these periods. His best driving occurred when listening to rock music.

On the other hand, the familiar music, that is similar to the relaxation music, helped to improve the reaction time.

The main limitation of this research is the complexity in the interpretation of the results. Some variables are easy to evaluate, such as the vehicle position in the lane, but many others can be quite complex, such as the eye tracker data.

The study of music effects on driving will never come to an unambiguous conclusion like "listen to rock music, you will not fall asleep". During this study, we confirmed the complicated relation between music and the behavior of the driver, most markedly in the case of the relaxation music - for most drivers it improved the reaction time, but for one driver it also increased the number of inappropriate steering actions. Not only that the same music has different effects on different individuals, but also it can have a both positive and negative effect on the same person.
The high individuality of the results is not necessarily a roadblock for the practical use of the achieved results. In everyday life we are using many devices that are calibrated for a particular person, such as mobile phones. In the automotive sector, we can imagine, a fatigue detector (using music as one of the inputs) that is calibrated for a particular driver, assuming that the car is usually driven by one person only, or, in the case of a fleet of cars or car-sharing, the driver-related data can be stored in the cloud.

\section{REFERENCES}

[1] Wang Dong-Yuan (Debbie), Jimison Zachary, Richard Dan, and Chuan Ching-Hua: EFFECT OF LISTENING TO MUSIC AS A FUNCTION OF DRIVING COMPLEXITY: A SIMULATOR STUDY ON THE DIFFERING EFFECTS OF MUSIC ON DIFFERENT DRIVING TASKS, PROCEEDINGS of the Eighth International Driving Symposium on Human Factors in Driver Assessment, Training and Vehicle Design, January 2015

[2] Steg Linda, Kai Epstude: The influence of music on mental effort and driving performance Ayc, a Berfu -nal*,Accid Anal Prev., 2012 September, DOI: 10.1016/j.aap.2012.01.022

[3] Brodsky, W.: The effects of music tempo on simulated driving performance and vehicular control. Transportation Research Part F, 4, (2002) pp. 219-241.

[4] Dalton, Brian H. Dalton, Gehm, David G..: Effects of noise and music on human and task performance: A systematic review. School of Human Kinetics and Recreation, Memorial University of Newfoundland, St. John's, Newfoundland, Canada, AlC 5S7. IOS Press. Occupational Ergonomics 7 (2007) pp. 143-152.

[5] Brown I. D.: Effect of a car radio on driving in traffic, Ergonomics 8 (1965), 475-479.

[6] Konz S. and McDougal D.: The effect of background music on the control activity of an automobile driver, Human Factors 10 (1968), pp. 233-244.

[7] Hammer S. E.: The effects of guided imagery through music on state and trait anxiety, Journal of Musical Therapy 33 (1996), pp. 47-70.

[8] Chaffin S. Roy M., Gerin W. and Christenfeld N.: Music can facilitate blood pressure recovery from stress, British Journal of Health Psychology 9 (2004), pp. 393-403. 
[9] Wisenthal D. L., Hennessy D. A. and Totten B .: The influence of music on mild driver stress, Journal of Applied Social Psychology 30 (2000), pp. 1709-1719.

[10] Wisenthal D. L., Hennessy D. A. and Totten B: The influence of music on mild driver aggression, Transportation Research Part F 6 (2003), pp. 125-134.

[11] Furnham A. and Bradley A.: Music while you work, The differential distraction of background music on the cognitive test performance of introverts and extroverts, Applied Cognitive Psychology 11 (1997), pp. 445-455.

[12] Aminudin M. and Nasir H.:Does Music Help to Stay Focus on the Road? WSEAS TRANSACTIONS on ACOUSTICS and MUSIC (2020), Vol 7, DOI: 10.37394/232019.2020.7.1

[13] Spurný, Andrš, Bouchner, Pučelík, Rokyta.: Testing a system for predicting microsleep, Lékař a technika. 2016, 46(2), 51-54. ISSN 0301-5491.

[14] Matthews, G. Quinn C.E.J. and Mitchell K.J. : Rock music, task-induced stress and simulated driving performance, in: Behavioural Research in Road Safety VIII, G.B. Grayson ed., Crowthorne, UK: Transport Research Laboratory, 1998, pp. 20-32.

[15] Beh, H. C. and Hirst R.: Performance on driving-related tasks during music, Ergonomic 42 (1999), pp. 1087-1098.

[16] Xu, J., Min, J., \& Hu, J. (2018). Real-time eye tracking for the assessment of driver fatigue. Healthcare Technology Letters, 5(2), 54-58. https://doi.org/10.1049/ht1.2017.0020.

[17] Li, R., Chen, Y. V., \& Zhang, L. (2019). Effect of Music Tempo on Long-Distance Driving: Which Tempo Is the Most Effective at Reducing Fatigue? I-Perception, 10(4). https://doi.org/10.1177/2041669519861982.

[18] Millet, B., Ahn, S., \& Chattah, J. (2019). The impact of music on vehicular performance: A meta-analysis. Transportation Research Part F: Traffic Psychology and Behaviour, 60, 743-760. https://doi.org/10.1016/j.trf.2018.10.007

\section{Creative Commons Attribution License 4.0 (Attribution 4.0 International, CC BY 4.0)}

This article is published under the terms of the Creative Commons Attribution License 4.0

https://creativecommons.org/licenses/by/4.0/deed.en_US 